\title{
Hardware and Software
}

\section{A floppy disk operating system for the 8080 and Z80}

\author{
KARL W. SCHOLZ \\ Advanced Interactive Systems, 8216 Pickering Street, Philadelphia, Pennsylvania 19150
}

\begin{abstract}
Techniques for the design and implementation of a floppy disk operating system for the $8080 \mathrm{~A}$ or $\mathrm{Z80}$ microprocessors are described. Implementation details are illustrated through reference to SOS, a prototype Small Operating System which runs with a single IBM-format floppy drive on an 8080. Techniques for floppy disk communication, disk directory design, and system resource management are described and illustrated.
\end{abstract}

For years, journals such as Behavior Research Methods \& Instrumentation have presented countless descriptions of the successes achieved by computerized research laboratories using equipment costing tens or hundreds of thousands of dollars. However, as both federal and institutional funds become increasingly difficult to obtain, a growing number of laboratories are forced to consider economic alternatives to total computerization, such as purchasing used equipment or microprocessor-based systems. Those who choose the microprocessor route quickly learn that, although inexpensive hardware is readily available, appropriate software is scarce and difficult to obtain. In a sense, the microprocessor user of today is in a position similar to that of the minicomputer user of a decade ago, in that both find the only practical source of appropriate software is internal. The present paper summarizes techniques central to the design of disk-based system software and illustrates these techniques using SOS, a Small Operating System, for the 8080 or Z80 microprocessor with at least $8 \mathrm{~K}$ of memory and one floppy disk.

\section{SYSTEM REQUIREMENTS}

A disk-based operating system should include as a minimum the following features. (1) A bootstrap loader, often ROM resident, to permit fast loading of the system when the machine is powered up. (2) Disk I/O primitives (e.g., subroutines) that permit reading from and writing to the disk. (3) A disk directory ("table of contents") through which any information stored on the disk can be located. (4) Utility functions for system and data file maintenance such as DIREC. TORY (display the "table of contents"), DELETE (to remove an unwanted data file), COPY (to copy an existing data file), RENAME (to alter the name of an existing data file), GET (to load a data file to memory), and SAVE (to create or update information on the disk from information in memory). (5) Utility programs such as a text editor, an assembler, and per- haps compiler(s) for higher level languages such as BASIC, PASCAL, or FORTRAN.

The list of desirable features can be greatly extended, resulting in the specification of a complex but comprehensive system. However, for a small research laboratory such a system can well represent an unnecessary extravagance. The following discussion will be restricted to the features listed above.

The development of the floppy disk is the single technological achievement which makes possible the creation of a functional but truly inexpensive laboratory computer system. In view of this, the following discussion centers primarily around techniques for organizing and communicating with a floppy disk.

\section{THE FLOPPY DISK AS A SECONDARY STORAGE MEDIUM}

A floppy disk is a disk of flexible Mylar $51 / 4$ or 8 in. in diameter which is coated with a magnetic emulsion similar to that on conventional recording tape. Disk drives are available for both sizes which provide either a single read-write head or multiple heads for double-sided recording; data can be written using either a single- or double-density format. Although double-density and double-sided formats are growing rapidly in popularity, the most widely used is the 8 -in. disk, single-density and single-sided. This type disk will be used in the following illustrations.

The disk's surface is formatted into a number of concentric "tracks," and each track is divided into a number of "sectors." In order to permit disk transportability from system to system, many manufacturers conform to a standard commonly referred to as the "IBM 3740" format, which provides 26 sectors of 128 bytes for each of 77 tracks. Disks formatted in this way provide 2,002 sectors, or 256,256 bytes of usable storage.

Disk drives are interfaced to the computer via a "controller" which attends to all the details of formatting, error detection, and parallel-serial conversion. Programming, therefore, requires positioning the disk 
head to the desired track, locating the desired sector, and transferring one or more sectors of information to or from the disk.

\section{THE DISK DRIVER}

The read and write routines (frequently referred to as the disk "driver") are central to the operation of any disk-based operating system. Since all disk I/O operations are mediated by the driver, it should be coded to maximize ease of use and should include facilities for error detection and recovery. Although the disk is accessed by both track number and sector number, the sector/track distinction should be of concern only to the driver, not to the user. A user need only specify a sector relative to the start of the disk or the start of a desired data file. The driver should calculate the correct track and sector within that track.

Dispite the extreme reliability of the floppy disk, errors occasionally occur. The IBM format includes several cyclic redundancy checks (CRCs, similar to a check sum) for each sector transfer. The controller generates a CRC when a sector is written and checks it when that sector is read. If the CRC check reveals an error, a status bit is set by the controller which is accessible to the software. Furthermore, the IBM format includes a sector header that precedes each sector and contains the correct track and sector address. On each transfer, the controller attempts to verify head position by inspecting this header, and sets an error status bit if the attempt fails.

Figure 1 illustrates the code in 8080 assembly language for a typical disk driver that includes error recovery procedures. It can be entered at either the READ or WRITE entry points, and expects to find the desired sector address (between 1 and 2,002) in the $\mathrm{BC}$ register, the desired number of sectors to be transferred in the DE register, and the memory address in the HL register. Although additional error processing can be included, this driver is entirely adequate for all but the most critical applications.

\section{DISK ORGANIZATION}

In the simplest case, disks serviced by a simple operating system need to be divided into three distinct sections: the system loader, the directory, and the user storage area. The term "system loader" refers to a simple routine stored in a known location on a disk (usually Track 0 , Sector 1) which on execution loads the monitor (the nucleus of the operating system). If the disk contains only the three sections mentioned. the system loader is required to search the directory for the name of the file containing the system monitor and then load it. For SOS, the system loader is con-

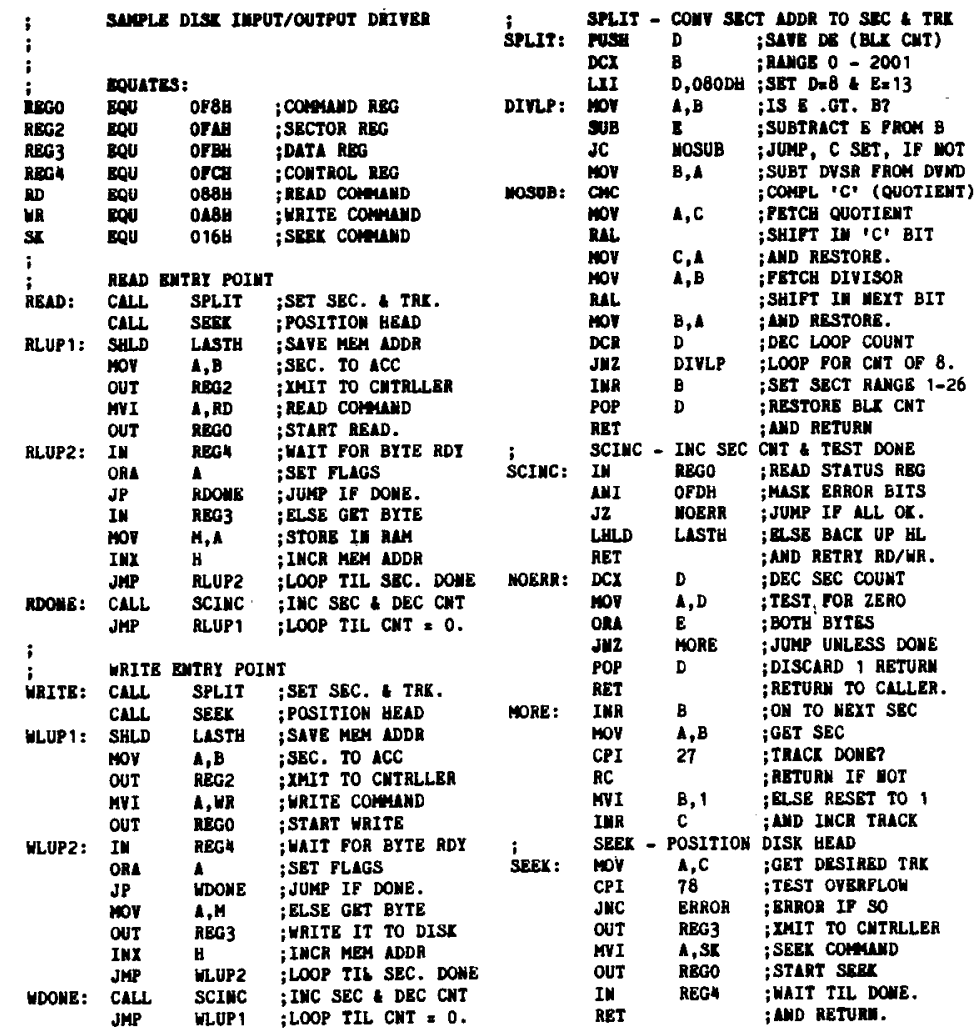

Figure 1. A sample floppy disk $\mathrm{I} / \mathrm{O}$ driver. The driver is entered with the desired disk sector in the $\mathrm{BC}$ register, the total sector count in the $\mathrm{DE}$ register, and the starting memory address in the $\mathrm{HL}$ register. 
siderably shortened by reserving a fourth distinct section of the disk for the monitor. The entire first track (26 sectors), minus the one sector assigned to the system loader, is reserved for this purpose. SOS reserves the next three tracks ( 78 sectors) for the directory and uses the remainder of the disk for file storage. On power-up, a ROM-resident bootstrap loads the system loader, which in turn loads the monitor and transfers control to it.

The disk directory is used as an index, or table of contents, through which files on the disk are located. A minimum disk system must retain at least the file name and the starting location (a sector address) in each directory entry. More complex systems also retain creation dates, last-access dates, file version numbers, file types, and additional relevant statistics for each file. In SOS, each directory entry contains the file name, its starting sector address, its size in blocks, its memory origin address, and (if the file is an executable program) its entry address. Files are named with a one- to six-letter file name and a two-letter file type (such as TX for text or DT for data).

For any disk system, it is essential that directory manipulation routines prohibit the accidental duplication of a file name and provide a means of managing disk space which becomes fragmented as old files are deleted and new ones are created. Among the most elegant methods for avoiding fragmentation is the technique of dynamically allocating disk sectors as needed, keeping track of their use with "bit maps" or linked lists. A second method involves creating a "dummy" directory entry for each sector or collection of sectors that has been freed by virtue of a file deletion. The third and simplest iechnique (used by SOS) is to periodically execute a "packing" routine that systematically collects all fragmented space and arranges it contiguously at the end of the disk. Packing need be performed only when required storage approaches the capacity of a disk pack.

SOS avoids both file name duplication and unnecessary fragmentation by performing two passes over the directory whenever a new file name is added. On the first pass, the entire existing directory is searched; if an old copy of the file name is located, it is deleted. On the second pass, the first area of the disk large enough to house the new file is used for its storage.

The system designer is cautioned to avoid attempting to save time by maintaining a portion of a disk directory or sector mapping information in RAM. Floppy controllers typically provide no mechanism for notifying a program if a disk is changed. Hence, it is possible to swap disks and create a situation where directory or sector mapping information for the wrong disk is in RAM, potentially leading to destruction of all information on the disk. Some commercially available operating systems have actually made this mistake.

\section{UTILITY FUNCTIONS}

Any disk operating system includes a collection of utility functions that perform necessary directory and file maintenance. Utilities fall into two general classes, those executed by keyboard command and those executed by call from a running program.

Keyboard-executable utilities presuppose the existence of a keyboard monitor that accepts and parses commands entered at the keyboard and directs them to the appropriate utility routines. For example, SOS accepts commands consisting of a directive followed by an optional list of arguments. The parser performs the following steps, in order. (1) The directive name is saved. (2) A pointer to the argument list is stored in a location accessible to any program. (3) A short table of utilities included in the monitor (GET, SAVE, and PATCH) is searched; if the directive is found, the appropriate utility is executed. (4) If the directive is not found in the monitor, the disk directory is searched for an executable program of that name; if found, it is loaded and executed. On execution, such programs can access the optional argument list by using the pointer saved in Step 2.

This technique has the advantage of providing extensibility. Any time a user requires an additional directive, he need only program the service routine for that directive and store it on the disk, and it effectively becomes a new SOS command. In operation, the distinction between monitor-resident and disk-resident utilities is transparent to the user. The SOS system includes a collection of frequently used disk-resident utilities coded in this manner (for directory listing, file deletion, file copying, file renaming, and disk packing).

The second class of utility functions are those accessible from within an executing program. As a bare minimum, a small operating system should provide facilities for program manipulation of disk files. In addition, a small system might include facilities for frequently used operations such as programmed timed delays, real-time clock support (for reaction time measurement), ASCII-decimal conversion, and any other operation that is unique to a particular application. SOS provides a carefully protected facility for program manipulation of disk files through three subroutine calls. The subroutine OPEN associates a file name with a "logical unit number" (an integer between 1 and 15). The subroutines READ and WRITE are used to transfer one or more sectors from/to the file associated with a particular logical unit number. The technique of associating a file name with a number and performaing disk $\mathrm{I} / \mathrm{O}$ with respect to the number is used frequently on larger systems. Among its many advantages, it permits the coding of a program without explicit knowledge of those files on which it will operate when executed. 
In SOS, file communication is mediated by a table referred to as a "file control block." The FCB is modified by the OPEN routine and utilized by the READ and WRITE routines. In both cases, the logical unit number is used to index the FCB. When OPEN is executed, it performs a directory search to locate the appropriate file. If so specified (by appropriate arguments to OPEN), a new empty file is created. In either case, the file's starting and ending sector addresses are inserted into the correct FCB entry for use by the READ and WRITE routines. FCB entry 0 is always open to the entire disk (for use by the system utility routines), thus leaving 15 logical unit numbers for use by user programs. Figure 2 illustrates the code used by the central disk driver to process all file references through the FCB. On entry, it assumes that the logical unit number is in the left 4 bits of the $\mathrm{BC}$ register, and the desired sector relative to the start of the file is in the remaining 12 bits. This routine is integrated into the SOS disk driver illustrated in Figure 1, beginning at the label SPLIT.

The complete SOS system includes three additional utilities: a text editor, an assembler, and a BASIC interpreter. These utilities have been coded to utilize the disk structure described above and to provide the tools necessary for most applications. All three utilities have been carefully optimized to use as little RAM as possible (the editor and assembler are each small enough to execute from a single $1 \mathrm{k}$-byte ROM). A detailed description of these three utilities is beyond the scope of this paper.

\section{SUMMARY}

We have attempted to summarize design considerations and implementation techniques for a small generalpurpose disk operating system. The techniques have

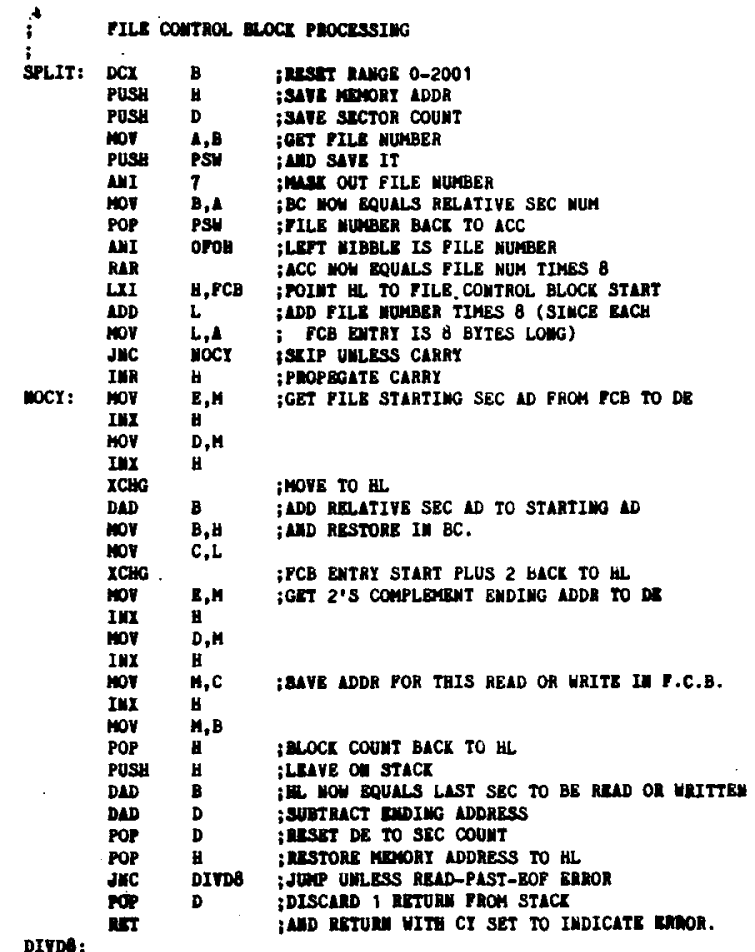

Figure 2. File control block processing. This routine, which is an optional component of the disk driver, processes a file number in the left 4 bits of the $\mathrm{BC}$ register. It expects to find the file control block beginning at symbolic location FCB.

been explored by implementing SOS, a small operating system for an 8080 or Z80 with at least $8 \mathrm{~K}$ of RAM and a single IBM-format floppy disk. Copies of the system have been evaluated on both 8080 and Z80 processors using the Tarbell disk controller, and, from all reports, it has served as an entirely adequate developmental vehicle despite its extreme simplicity.

(Accepted for publication June 13, 1978.) 